\title{
EDITORIAL AND COMMENT
}

\section{Experiences with POLST: Opportunities for Improving Advance Care Planning

\author{
Editorial \& Comment on: "Use of Physician Orders for Life-Sustaining Treatment among \\ California Nursing Home Residents"
}

\author{
Ursula K. Braun, MD, MPH
}

Department of Medicine, Section of Geriatrics, Baylor College of Medicine, Center for Innovations in Quality, Effectiveness and Safety, Michael E. DeBakey VA Medical Center, Houston, TX, USA.

J Gen Intern Med 31(10):1111-2

DOI: $10.1007 / \mathrm{s} 11606-016-3795-y$

(c) Society of General Internal Medicine 2016

$\mathrm{P}$ hysician Orders for Life-Sustaining Treatment (POLST), sometimes also known under other terms, e.g. Medical Orders for Life-Sustaining Treatment (MOLST), began in Oregon in 1991 as the "POLST Paradigm Initiative", a task force of healthcare professionals and ethicists from the Center for Ethics in Health Care at Oregon Health \& Science University (OHSU). The goal was to facilitate patients' choices regarding end-of-life (EOL) care, in particular life-sustaining medical treatments. The "paradigm" was designed around a process of EOL counseling that would lead to completion of a POLST form. In 2004, OHSU's Center for Ethics in Health Care assembled a task force of representatives from participating states to facilitate the propagation of the POLST paradigm nationally. The new National POLST Paradigm Task Force (NPPTF) has established standards by which individual states can develop "endorsed" POLST programs. POLST forms are now used in over 40 states. In 19 of these, the NPPTF has endorsed state POLST programs as they have become standard components of advance care planning in their location, have addressed legal and regulatory issues, and have developed strategies for ongoing implementation and quality assurance. ${ }^{1}$ In California, the state about which Jennings et $\mathrm{al}^{2}$ report in this issue, POLST was fully implemented only in 2009, but is considered (along with Oregon and West Virginia) to have a 'mature' POLST program by the NPPTF based on POLST use by $50 \%$ or more of hospitals, nursing homes, and hospices in each region of the state.

POLST is a standardized, portable, single-page, brightly colored and thus highly visible document, with the goal of translating a discussion between a patient and/or his/her surrogate decision-maker and a physician into a medical order set

The content of this work does not represent the views of the Department of Veterans Affairs or the United States Government.

Published online July 11, 2016 that is legal across not just one care setting but across the continuity of possible care settings a patient may experience within a single state. It can be used, for example, by paramedics, fire departments, and police, in hospitals, and in residential care facilities to provide the treatment a patient wants. POLST can help reduce unwanted hospitalizations and honor a patient's end-of-life wishes. To determine whether a POLST form should be completed, clinicians are encouraged to ask themselves the 'surprise question', "Would I be surprised if this person died in the next year?" If the answer is 'No', initiating a POLST form is considered appropriate. The POLST concept was developed with terminally or chronically ill frail elderly patients in mind, though legally many states do not limit its use to certain patient groups.

POLST was found to be an effective tool in studies that examined whether treatments provided at the end of life matched the orders on the form, ${ }^{3-5}$ though far less research has been done to evaluate whether POLST orders truly reflect patient wishes; this is an important area where further research would be useful. ${ }^{6}$ Additionally, the TRIAD (The Realistic Interpretation of Advance Directives) studies showed misunderstandings among various healthcare workers regarding the interpretation of advance directives and related documents. TRIAD VII specifically examined pre-hospital providers' interpretation of POLST in Pennsylvania, a state with an NPPTF-endorsed POLST program. Emergency personnel's interpretation of the form varied considerably, except for the 'full code' scenario, raising patient safety concerns regarding both under- and over-treatment. ${ }^{7}$ While POLST forms can be effective advance care planning tools, they are different from advance directives: Advance directives express preferences for desired and undesired treatments, and are completed by patients, not by surrogates - in fact, they cannot be completed by patients once they have lost decision-making capacity. A POLST form, however, similar to an out-of-hospital do-not-resuscitate $(\mathrm{OOH}-$ DNR) form, can be completed with surrogates, and because it turns treatment preferences into a medical order, it is actionable and binding. Additionally, because it follows patients through their care settings, it is much more likely to be followed than advance directives, which can sometimes be ambiguous, requiring interpretation by surrogates. Advance directives may also not always be readily available — especially not across new care settings. Many 
organizations, including the National Quality Forum, the National Academy of Sciences Institute of Medicine, the American Academy of Hospice and Palliative Medicine, the Society for PostAcute and Long-Term Care Medicine (AMDA), the American Nurses Association, and the National Association of Social Workers, support the POLST paradigm; on the other hand, it has been criticized by the Catholic Medical Association. (Neither the Society of General Internal Medicine nor the American Geriatrics Society has passed formal resolutions regarding POLST).

POLST forms are binding medical orders. While this is their biggest advantage, this may also be a weakness raising ethical concerns, especially if the informed consent process leading to POLST is unclear. Not all states require signatures by the patient or the designated surrogate.

In this issue of JGIM, Jennings et al. ${ }^{2}$ report on the use of POLST among California nursing home residents in 2011, just 3 years after state-wide implementation. Beyond the large sample of nursing homes and patients, one of the biggest strengths of their study is that California is the - so far only — state that collects information about POLST in their Minimum Data Set(MDS) Section S. The MDS is a mandatory survey that collects information on all nursing home residents, and thus the authors were able to obtain complete records.

Some of the results were expected: longer-stay residents had much higher completion rates for POLST than short-stay residents, and the rate of POLST completion increased over time. Explaining the wide variation in POLST use at the nursing home level seems more difficult: in $40 \%$ of nursing homes most residents had a POLST, while in $20 \%$ of nursing homes very few residents had a POLST. Only $26 \%$ of nursing home residents with a POLST also had an advance directive. This could reflect the view that advance directives are superfluous when POLST is available, or there may be other reasons. Cognitively impaired residents were less likely to have completed a POLST than cognitively intact residents, which likely reflects greater uncertainty on the part of their surrogates regarding the residents' supposed wishes for future care. However, it was quite astonishing that no ethnic or racial variations were found in POLST completion rates, given that multiple studies have shown that minorities are much less likely to have advance directives or living wills - which POLST approximates to some degree - and despite many studies showing that minorities receive more aggressive end-of-life care. The authors speculate that this might be due to POLST's broad-based statewide public health intervention, combined with a strong grassroots initiative, though much further exploration may be needed.

Another surprising finding was that $13 \%$ had invalid POLST forms due to missing physician signatures. Since POLST forms are a medical order, they must always be signed by a physician (though nurse practitioners or physician assistants in many states - and in California since 2016 - can also sign these forms) and, depending upon the state, by the patient or surrogate (California requires either a patient or surrogate signature). An order as important as POLST should have a carefully documented informed consent process. While facilitators such as social workers and nurses are certainly helpful in explaining the form and its purpose, the role of the physicians would be marginalized if they were not the ones leading this communication process. It is unclear why so many forms lacked physician signatures, and this was not further discussed by the authors: could it be that the forms are being used in discussions with patients and/or surrogates by non-physicians, e.g. social workers or directors of nursing, and are later being presented to physicians for signature? This would be of concern in situations in which the details of a resident's medical condition were not fully understood by other members of the healthcare team. Future research should examine the actual decision-making process leading up to a completion of POLST and ways to better ensure high quality in this process.

The article by Jennings et al. is especially timely given the recent decision by the Centers for Medicare \& Medicaid Services to compensate physician efforts to have end-of-life care discussions for advance care planning with their patients. We should therefore expect the rates for POLST completion to further increase in states where they are available, and hope that forms with missing physician signatures will decrease. Quality improvement and patient safety initiatives should focus on ways to measure the quality of such end-of-life discussions that culminate in POLST completion.

Corresponding Author: Ursula K. Braun, MD, MPH; Department of Medicine, Section of Geriatrics, Baylor College of Medicine, Center for Innovations in Quality, Effectiveness and SafetyMichael E. DeBakey VA Medical Center, 2002 Holcombe Blvd, VAMC 152, RECL, Houston, TX 77030, USA (e-mail: ubraun@bcm.edu).

\section{Compliance with Ethical Standards:}

Conflict of Interest: I have no conflicts of interest.

Funding: This work was supported by the Houston VA HSR\&D Center for Innovations grant (CIN 13-413).

\section{REFERENCES}

1. National POLST Paradigm www.polst.org last Accessed 6/1/2016.

2. Jennings LA, Zingmond D, Louie R, Tseng C, Thomas J, O'Malley $K$, Wenger NS. Use of Physician Orders for Life Sustaining Treatment among California Nursing Home Residents. J Gen Intern Med. 2016. doi:10.1007/s11606-016-3728-9.

3. Tolle S, Tilden VP, Dunn P, Nelson C. A prospective study of the efficacy of the physician orders for life-sustaining treatment. J Am Geriatr Soc. 1998;46(9): 1097-1102.

4. Hickman S, Tolle S, Brummel-Smith K, Carley MM. Use of the physician orders for life-sustaining treatment program in Oregon nursing facilities: beyond resuscitation status. J Am Geriatr Soc. 2004;52(9):1424-1429. doi:10.1111/j.1532-5415.2004.52402.x.

5. Fromme EK, Zive D, Schmidt TA, Cook JNB, Tolle sw. Association between physician orders for scope of treatment and in-hospital death in Oregon. J Am Geriatr Soc. 2014;62(7):1246-1251.

6. Meyers JL, Moore C, McGrory A, Sparr J, Ahern M. Use of the Physician Orders for Life-Sustaining Treatment (POLST) form to honor the wishes of nursing home residents for end of life care: preliminary results of a Washington state pilot project. J Gerontol Nurs. 2004;30:37-46.

7. Mirarchi FL, Cammarata C, Zerkle SW, Cooney TE, Chenault J, Basnak D. TRIAD VII: do prehospital providers understand Physician Orders for LifeSustaining Treatment documents? J Patient Saf. 2015;11(1):9-17. doi:10. 1097/PTS.0000000000000164. 